\title{
Stress Coping and Resilience in College Students with Depression
}

\author{
Dahni Kim, MD, Bo-Hyun Yoon, MD, PhD, Young-Hwa Sea, MD, Hangoeunbi Kang, MD, \\ Kyungmin Kim, MD, Jye-Heon Song, MD, Suhee Park, MD \\ Department of Psychiatry, Naju National Hospital, Naju, Korea
}

Background: Depression is increasing among college students in general. Moreover, almost one-third of college students have been reported to suffer from depression. Thus, this study aimed to assess differences in stress coping strategies and resilience between depressed and normal-mood groups among college students.

Methods: A total of 3,306 college students participated in this study. The students responded to a questionnaire that included questions of the Center for Epidemiologic Studies Depression (CES-D) scale, stress coping scale (SCS), and brief resilience scale. Logistic regression analysis was conducted to evaluate the impact of variables on depression.

Results: Using the CES-D (cutoff score $\geq 21$ ), 423 (12.8\%) college students were classified as depressed. Adjusting for individual demographic factors, the SCS results of the students with depression showed significantly higher scores in emotion-focused coping $(p<0.001)$, wishful thinking $(p<0.001)$, and lower problem-focused coping $(p<0.001)$ than the normal-mood group. Moreover, they presented lower resilience scores. Students who had emotion-focused coping (odds ratio $[O R], 1.11 ; p<0.001)$ and lower resilience scores $(O R, 0.76 ; p<0.001)$ were associated with higher CES-D scores.

Conclusion: The study findings revealed significant differences between the depressed and normal-mood groups in terms of stress coping skills and resilience, suggesting the need for promoting stress coping strategies and resilience to lower depression-related problems among college students.

Keywords Depression; Coping skills; Resilience, psychological; Health services, student

\section{INTRODUCTION}

Depression presents symptoms, such as depressed mood, diminished interest, decreased weight or appetite, insomnia, loss of energy, and poor concentration [1], which result in damages in various areas of life [2]. In particular, if the first episode occurs during college years, careful monitoring is essential because the impact may extend to a future occupation and environment due to functional impairment and result in significant changes in an individual's life [3]. As reported by meta- studies, the prevalence of depression among college students in the U.S. varies from $10 \%$ to $85 \%$ [2], and the prevalence in Korean college students has been reported to be between $7.7 \%$ and $14.9 \%$ in studies conducted with college students in the Jeju or Gwangju-Jeollanam-do regions $[4,5]$. The incidence of depression is increasing every year among college students; it is worthwhile to note that, according to a meta-study, one-third of college students report difficulties with depressive symptoms [2]. According to data published by Boston University in 2021, a questionnaire conducted with 33,000 U.S. col-

Received October 1, 2021, Revised October 28, 2021, Accepted November 5, 2021

Correspondence: Bo-Hyun Yoon, MD, PhD

Department of Psychiatry, Naju National Hospital, 1328-31 Senam-ro, Sanpo-myeon, Naju 58213, Korea

TEL +82-61-330-4102 FAX +82-61-330-4155 E-mail yoonbh@chollian.net ORCID https://orcid.org/0000-0002-3882-7930

Co-Correspondence: Young-Hwa Sea, MD

Department of Psychiatry, Naju National Hospital, 1328-31 Senam-ro, Sanpo-myeon, Naju 58213, Korea

TEL +82-61-330-4151 FAX +82-61-330-4150 E-mail yhsea1414@hanmail.net ORCID https://orcid.org/0000-0002-0420-2609

Copyright $\odot$ by Korean Society for Affective Disorders.

This is an Open Access article distributed under the terms of the Creative Commons Attribution Non-Commercial License (http://creativecommons.org/licenses/ by-nc/4.0/) which permits unrestricted non-commercial use, distribution, and reproduction in any medium, provided the original work is properly cited. 
lege students during the fall semester in 2020 found that $39 \%$ of students reported depressive symptoms, urging the faculty and the colleges to take actions [6]. In Korea, depressive symptoms among college students are becoming increasingly important as well, and the government has announced the expansion of support for college counseling centers [7].

While there are various causes reported for the onset of depression, the general hypothesis is that it is triggered by the psychosocial stress(es) of an individual who has a biological predisposition for susceptibility [8]. The concept of stress evolves from a simple concept of a changed psychophysiological state to a threat to psychological well-being beyond an individual's capacity in a changing environment [9]. Each individual has a different tolerance for stress, even in the same situation, and they focused on how an individual uses different stress coping strategies in a stressful situation [9]. Stress coping strategies can be grouped into different categories; namely, there are the problem-focused coping strategies that focus on active resolution of the problem, emotionfocused coping strategies that aim to resolve emotional tension regarding the stressful incident, and avoidance [10]. In Korea, the strategies were largely grouped into active coping and passive coping [11]. Active coping includes attempts at problem-focused coping and seeking social support, whereas passive coping includes emotion-focused strategies and wishful thinking [10]. Although stress coping strategies largely focus on helping individuals handle stressful situations in a more adaptive way, previous research has found that they are associated with depressive symptoms [8]. Depressed groups are more likely to engage in emotion-focused coping, while normal groups tend to utilize problem-focused coping [8].

In addition to the strategies for coping with depression, we consider the concept of resilience in a similar context; the concept was introduced based on the observation that there were differences in the way individuals recovered psychologically after experiencing similar situations involving adversity or danger [12]. Resilience assesses the ability of an individual to cope with a difficult situation and is regarded as a protective factor against depression [13]. Although stress coping strategies and resilience are related factors, they have different characteristics; stress coping strategies refer to intentional action taken to cope with the stress whereas resilience is the successful use of the stress coping strategy [14].
Based on the above definitions, the present study aims to survey depression in college students in Gwangju and Jeollanam-do regions of Korea. From this study, we aimed to evaluate factors associated with depression and assess their stress coping strategies as well as resilience to examine the interrelations among depression, stress coping strategies, and resilience.

\section{MATERIALS AND METHODS}

\section{Study subjects}

This study was conducted through 3,569 college students enrolled in 13 colleges in Gwangju and Jeollanamdo regions between March 2019 and June 2019. All participants signed on the consent form after prior explanation about the purpose of the study. They were then asked to complete a self-reported questionnaire. The analysis was conducted based on the responses of 3,306 subjects after excluding 263 subjects (7.4\%) who did not complete the questionnaire provided. The study was approved by the Institutional Review Board of the Naju National Hospital (NNH-HR-2021-1).

\section{Assessment tools}

\section{1) Self-report questionnaire}

Sociodemographic information sought from the subjects included sex, grade, height, weight, place of birth, college major, living arrangement, marital status of parents, and the subject's self-perceived socioeconomic level, alcohol consumption, smoking history. The questionnaire also collected information on the history of suicidal ideation, suicide attempts existed on the past or still in the present.

\section{2) Center for epidemiologic studies depression (CES-D) scale}

The CES-D Scale published by the U.S. National Institute of Mental Health was translated into Korean and used to assess the severity of depression. On this scale, each depressive symptom is rated from 0 to 3 for all the 20 items. In a study that examined the validity of the Korean version of the CES-D scale, score more than 21 was presented as the ideal threshold score for screening depressive symptoms [15]. Therefore, those who scored more than 21 were considered as a depressed group in 
this study.

\section{3) Stress coping scale (SCS)}

The stress coping strategy scale used in this study was based on the 68 -item questionnaire published by Lazarus and Folkman [9], which was subsequently revised to a 62 -item questionnaire with two categories, active coping and passive coping, by Kim [11] after factor analysis. Active coping includes problem-focused coping, in which an individual actively engages to confront and resolve the problem, seeking social support, recognizes the difficulty they are facing, and requests for help from others to deal with the stress [11]. Passive coping involves wishful thinking, in which an individual tries to change their way of thinking rather than recognizing the difficulty or the problem all the while pretending as if it has no relevance to them. Emotion-focused coping is also one of passive coping, in which the individual engages in relieving emotions or examines ways to change their thinking rather than change the circumstances. [11]. subsequently, studies were conducted using a 24 -item questionnaire in continuation of the 4 factors identified by Kim [11], and this study also used the 24-item questionnaire revised by Park [10]. The reliability of the scale in this study as evaluated by Cronbach $\alpha$ was 0.79 for the total score, and $0.80,0.68,0.78$, and 0.70 for problem-focused coping, seeking social support, wishful thinking, and emotion-focused coping, respectively.

\section{4) Brief resilience scale (BRS)}

BRS, a scale configured to measure the specific resilience in groups [16], is one of the four main scales used to measure resilience [17]. The scale comprises 6 questions in all, 3 affirmatory and 3 negatory. Each item is scored on a scale of 1 to 5 points, and the responses to negatory questions were calculated inversely so that a higher total score indicated a higher resilience.

\section{Statistical analysis}

First, the CES-D score of 21 was set as the cut-off point for categorizing the students into the depressed and non-depressed group. A $\chi^{2}$ test was conducted to analyze the differences in grade, sex, marital status of parents, socioeconomic status, suicidal ideation, and history of suicide attempts, which were reported as significantly associated with depression in previous research $[2,4,5]$. Next, the differences in the stress coping strategies and resilience were analyzed using an independent $\mathrm{t}$-test. This was followed by a logistic regression analysis to examine the differences in the depressed group using the CES-D score based on the stress coping strategy after controlling for the sociodemographic variables that showed a difference between the two groups. Data analysis was performed using IBM SPSS Statistics for Windows, Version 21.0 (IBM Corp., Armonk, NY, USA). The statistical significance level in this study was $\mathrm{p}<0.05$ for all analyses.

\section{RESULTS}

\section{Sociodemographic characteristics}

Among the 3,306 college students, 423 subjects $(12.8 \%)$ were categorized as the depressed group and 2,883 subjects $(81.2 \%)$ were in the non-depressed group. Significant differences were found between the two groups depending on grade, sex, marital status of the parents, socioeconomic status, suicidal ideation, and history of suicide attempts (Table 1).

\section{Factors associated with depression}

The variables that showed a statistically significant difference on the CES-D scale as the results of $\chi^{2}$ test and the independent $\mathrm{t}$-test were grade, sex, marital status of parents, socioeconomic status, suicidal ideation, and suicidal attempt, in addition to problem-focused coping, seeking social support, emotion-focused coping, wishful thinking, and resilience. To identify their impact on the severity of depression measured by the CES-D, these were set as independent variables while the positivity of CES-D was used as the dependent variable to conduct a logistic regression analysis. Logistic regression analysis was conducted by excluding suicide attempt due to the potential problem of multicollinearity that could emerge from suicidal ideation and previous suicide attempts. This is because suicide ideation was considered more a part of the chain of processes starting from suicide ideation, suicide plans, suicide attempts, and suicide [18]. Therefore, when surveying depressive symptoms for screening, it was already assumed that suicidal ideation itself is sufficiently correlated with depressive symptoms [18].

The results of the logistic regression analysis showed 
Table 1. General characteristics of the depressed and non-depressed college students

\begin{tabular}{|c|c|c|c|c|}
\hline Variable & Depressed & Non-depressed & Total & $\mathrm{p}$-value \\
\hline Total & $423(12.8)$ & $2,883(81.2)$ & $3,306(100)$ & \\
\hline Grade & & & & $<0.001$ \\
\hline Freshman & $239(11.1)$ & $1,914(88.9)$ & $2,153(65.1)$ & \\
\hline Sophomore & $96(16.6)$ & $483(83.4)$ & $579(17.5)$ & \\
\hline Junior & $46(12.5)$ & $321(87.5)$ & $367(11.1)$ & \\
\hline Senior & $42(20.3)$ & $165(79.7)$ & $207(6.3)$ & \\
\hline Sex & & & & $<0.001$ \\
\hline Male & $122(28.8)$ & $1,223(42.4)$ & $1,345(40.7)$ & \\
\hline Female & $301(71.2)$ & $1,660(57.6)$ & $1,961(59.3)$ & \\
\hline Marital status of parents & & & & $<0.05$ \\
\hline Married & $336(79.4)$ & $2,462(85.4)$ & $2,798(84.6)$ & \\
\hline Separated $^{a}$ & $87(20.6)$ & $421(14.6)$ & $508(15.4)$ & \\
\hline Self-perceived socioeconomic status & & & & $<0.001$ \\
\hline High & $28(6.6)$ & $275(9.5)$ & $303(9.1)$ & \\
\hline Medium & $297(70.2)$ & $2,264(78.5)$ & $2,561(77.5)$ & \\
\hline Low & 98 (23.2) & $344(12.0)$ & $442(13.4)$ & \\
\hline Suicidal ideation & & & & $<0.001$ \\
\hline Yes & $224(52.9)$ & $577(20.0)$ & $801(24.2)$ & \\
\hline No & $199(47.1)$ & $2,306(80.0)$ & $2,505(75.8)$ & \\
\hline Suicidal attempt & & & & $<0.001$ \\
\hline Yes & 75 (17.8) & $104(3.6)$ & $179(5.4)$ & \\
\hline No & 348 (82.2) & $2,779(96.4)$ & 3,127 (94.6) & \\
\hline
\end{tabular}

Values are presented as number (\%).

ancludes divorced and bereaved.

that depressive symptoms were lower with statistical significance for male subjects (odds ratio [OR], 0.77; 95\% confidence interval [CI], 0.59-0.99; $\mathrm{p}<0.05)$ of medium range as opposed to low socioeconomic status (OR, 0.67; 95\% CI, 0.49-0.91; $\mathrm{p}<0.05)$. In contrast, depression was more likely when there was suicidal ideation (OR, 2.44; 95\% CI, 1.91-3.11; $\mathrm{p}<0.001)$ and when the subjects were more likely to use emotion-focused coping (OR, 1.11; 95\% CI, 1.06-1.16; $\mathrm{p}<0.001)$ with lower resilience (OR, 0.76; 95\% CI, 0.73-0.79; $<<0.001)$. Meanwhile, statistically significant difference was not seen among those with grades $(p=0.064)$ married parents $(p=0.473)$, with high as opposed to low socioeconomic status $(p=0.413)$, problem-focused coping ( $\mathrm{p}=0.293)$, seeking social support $(\mathrm{p}=0.487)$, and wishful thinking $(\mathrm{p}=0.056)$ (Table 1,3$)$.

\section{Differences in stress coping strategies}

The depressed and non-depressed groups were compared by dividing the stress coping strategies into active versus passive coping. The depressed group showed sta- tistically significant lower scores for active coping with statistically significant high scores for passive coping $(\mathrm{p}<0.05)$. Next, active coping was divided into problemfocused coping and seeking social support while passive coping was divided into emotion-focused coping and wishful thinking. The depressed group was less likely to engage in problem-focused coping and more likely to engage in seeking social support, emotion-focused coping, and wishful thinking $(\mathrm{p}<0.05)$. Logistic regression analysis showed that depression was more likely when the subjects use emotion-focused coping (OR, 1.11; 95\% CI, 1.06-1.16; p<0.001) (Table 2, 3).

\section{Differences in resilience}

When compared using the resilience scale, the depressed group was found to have a significantly lower resilience with a score of $15.99 \pm 4.05$ as opposed to $20.95 \pm 4.20$ for the normal group $(\mathrm{p}<0.001)$. The result of logistic regression analysis showed that depression was associated with lower resilience (OR, 0.76; 95\% CI, 0.73 - 
Table 2. Differences in stress coping and resilience between the depressed and the non-depressed college students

\begin{tabular}{|c|c|c|c|c|}
\hline Variable & Depressed & Non-depressed & $\mathrm{t}$ & $p$-value \\
\hline Active coping $^{a}$ & $28.35 \pm 5.49$ & $29.19 \pm 6.12$ & 1.65 & $<0.05$ \\
\hline Problem-focused coping & $13.95 \pm 3.54$ & $14.94 \pm 3.69$ & 4.82 & $<0.001$ \\
\hline Seeking social support & $14.62 \pm 2.82$ & $14.24 \pm 3.23$ & -2.45 & $<0.05$ \\
\hline Passive coping $^{a}$ & $29.92 \pm 4.93$ & $28.20 \pm 5.60$ & -6.79 & $<0.001$ \\
\hline Emotion-focused coping & $13.36 \pm 2.88$ & $12.84 \pm 2.99$ & -4.07 & $<0.001$ \\
\hline Wishful thinking & $16.56 \pm 3.14$ & $15.36 \pm 3.48$ & -7.60 & $<0.001$ \\
\hline Resilience $^{b}$ & $15.99 \pm 4.05$ & $20.95 \pm 4.20$ & 23.33 & $<0.001$ \\
\hline
\end{tabular}

Values are presented as mean \pm standard deviation.

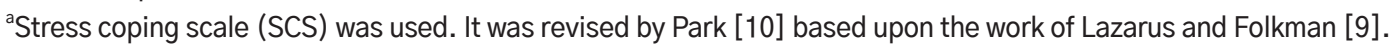

${ }^{\mathrm{b}}$ Brief resilience scale (BRS) was used [16].

Table 3. Factors associated with depression in college students

\begin{tabular}{|c|c|c|c|c|c|}
\hline \multirow{2}{*}{ Variable } & \multicolumn{5}{|c|}{ Depressed } \\
\hline & $\mathrm{B}$ & SE & OR & $95 \% \mathrm{Cl}$ & $p$-value \\
\hline Grade & 0.12 & 0.06 & 1.12 & $0.99-1.27$ & 0.064 \\
\hline Sex, male & -0.26 & 0.13 & 0.77 & $0.59-0.99$ & $<0.05$ \\
\hline Marital status of parents, married & -0.11 & 0.16 & 0.89 & $0.65-1.22$ & 0.473 \\
\hline SES, high (ref: low) & -0.22 & 0.27 & 0.79 & $0.47-1.37$ & 0.413 \\
\hline SES, medium (ref: low) & -0.41 & 0.16 & 0.67 & $0.49-0.91$ & $<0.05$ \\
\hline Suicidal ideation & 0.89 & 0.12 & 2.44 & $1.91-3.11$ & $<0.001$ \\
\hline Problem-focused coping & -0.02 & 0.02 & 0.98 & $0.94-1.02$ & 0.293 \\
\hline Seeking social support & -0.02 & 0.03 & 0.98 & $0.93-1.04$ & 0.487 \\
\hline Emotion-focused coping & 0.10 & 0.02 & 1.11 & $1.06-1.16$ & $<0.001$ \\
\hline Wishful thinking & 0.05 & 0.03 & 1.05 & $0.99-1.10$ & 0.056 \\
\hline Resilience & -0.28 & 0.09 & 0.76 & $0.73-0.79$ & $<0.001$ \\
\hline
\end{tabular}

SES, self-perceived socioeconomic status; $B$, unstandardized beta coefficients; SE, standard error; OR, odds ratio; Cl, confidence interval.

OR was calculated using a logistic regression analysis.

$0.79 ; \mathrm{p}<0.05)$ (Table 2, 3).

\section{DISCUSSION}

In this study conducted on college students from Gwangju and Jeollanam-do regions, the CES-D scale was used to identify the depressed group of students and the factors relating to depression were analyzed. Among the total number of participants $(n=3,306), 12.8 \%$ was found to be in the depressed group. A previous study conducted with 1,205 students in Jeju region reported $7.7 \%$ prevalence of depressive symptoms as measured by Beck depression inventory (BDI) [4]. Another study conducted with the 1,730 college students in the Honam region reported a prevalence of $14.9 \%$ when measured using BDI [5]. This is much higher than the lifetime prevalence of $5.0 \%$ and annual prevalence of $1.5 \%$ reported by a survey on mental illness conducted by the Ministry of Health and Welfare (2016) to examine the prevalence of depression in Korean adults [19]. This difference can be attributed to the fact that, while the studies mentioned above used self-report questionnaires, the Korean version of the Composite International Diagnostic Interview was administered by an employee trained in mental illness survey. According to a study that analyzed 24 papers published for 20 years since 1990 , the prevalence of depression ranged from $10 \%$ to $85 \%$ in college students [2]. Despite such variance in the reported prevalence of depression in college students, depression among college students is a common health issue [20], and the trend is clear as the meta-study con- 
ducted in 2013 and 2016 reported a continuous increase in the prevalence $[2,21]$.

Logistic regression analysis conducted on factors related to depression showed that the severity of depression was lower in male than in female; likewise, it was lower among those whose socioeconomic status was in the medium range as opposed to those with a low socioeconomic status. Depressive symptoms were found to be associated with suicidal ideation, emotion-focused coping and low resilience.

Sex difference was found to affect depression. male were less at risk compared to female; this is consistent with the findings of the mental health survey conducted by the Ministry of Health and Welfare in 2016, wherein major depressive disorder was higher in female $(6.9 \%)$ compared to that in male (3.0\%) [19]. This could be explained by two factors: the psychosocial factor of women experiencing high levels of daily stress for performing their socially required role with limited outcomes [22] and the reported biological factor of susceptibility to depression in women based on the interaction of estrogen and serotonin [23].

In the present study, socioeconomic status was also associated with depressive symptoms among college students. The prevalence of depression was lower among individuals of medium socioeconomic status than among those of low socioeconomic status. In previous studies, depression and suicidal ideation increased when the perceived economic stress was high in college students [24]. There was also a finding that depression is related to low socioeconomic status in Finland, Poland, and Spain [25]. However, in this study, there was no significant difference between individuals of high socioeconomic status and those of low socioeconomic status; future studies are recommended to conduct in-depth investigations on this.

Suicide is a very serious matter to consider for individuals with depression. According to the Suicide Prevention Manual released by the Central Suicide Prevention Center in 2021, the prevalence of suicide in 2019 was 26.9 per 100,000 people - a 0.2 increase $(0.9 \%)$ from the previous year and the highest among OECD countries [26]. In this study, there were significant differences in suicidal ideation and suicidal behaviors between individuals with and without depression. According to Statistics Korea, suicide rates between ages 20 and 29 years increased from $17.6 \%$ in 2018 to $19.2 \%$ in 2019 [27]. Specifically, in 2020, women in their 20s accounted for the highest proportion (32.1\%) of all suicide cases in Korea [27]. Suicidal ideation increases with depressive symptoms, and depressive symptoms may in turn be deteriorated by suicidal ideation [28]; thus, it is critical to promptly identify students exhibiting depressive symptoms, to provide timely mental health care.

In this study, the depressed group was more likely to engage in emotion-focused coping. This is partially consistent with the findings from a study by Orzechowska et al. [8] that depressed individuals are more likely to use emotion-focused coping while non-depressed individuals tend to use active coping [8]. The present study's results also corroborate Lee and Kim's [29] findings that the depressed individuals are more likely to use passive coping, including emotion-focused coping, and less likely to use active coping [29]. Furthermore, one study showed that depression is aggravated regardless of active coping when passive coping with emotion-focused coping is used more frequently [29]; this is also consistent with this study's findings. It is known that the relationship between stress coping strategies and depression is mutually affected [8]. Maladaptive stress coping strategies tend to increase the incidence of depressive symptoms, which may reduce one's coping ability to manage stress when depression occurs [8]. This could lead to reduced quality of life and deterioration of health. Thus, considering previous findings that interventions for passive coping improved depressive symptoms [30], enhancing stress coping strategies for college student may reduce depression.

In this study, the depressed group reported low resilience. Resilience has a positive effect on mental health by providing psychological homeostasis when faced with unpredictable stress; it enhances adaptability to situations and acts as a protective factor against depression [31]. Southwick and Charney [32] summarized and reported the effects of various neurotransmitters on resilience and depression. Both human and animal studies have shown behaviors predominantly observed in depression, such as decreased effort or becoming passive in changing the negative experiences, when faced with intolerable stress due to lack of resilience; this in turn reduces proliferation of cells in the hippocampus and increases the feeling of helplessness [32]. Normal noradrenergic activity range maintains one's resilience to stress, and adequate dopaminergic activity can also help increase resilience [32]. There are also psychosocial factors related to resilience; these include having 
someone who can provide care, previous experience of overcoming adversity, cognitive flexibility, ability to perceive situations optimistically, ability to regulate emotions, excellent self-efficacy, strong social support system, and continued exercise, which can be included in a depression prevention program for college students [32]. Resilience has many ways of components but generally categorized to three factors which are the ability to regulate one's emotion, receive help from others.

Although not presented in the results, when the interaction between stress coping strategies and resilience was examined using multicollinearity, there was no interaction between stress coping strategies and resilience. This is consistent with a study on elementary school students that reported no significant correlation among the sub-factors when stress coping strategies and resilience were examined [33]. In contrast, a study examining resilience and stress coping strategies in college students reported a correlation between problem-focused coping, seeking social support and resilience [34], and another study on nurses showed a significant relationship with problem-focused coping and resilience [35]. However, according to existing literature, stress coping strategies refer to using adaptive skills for managing stress, whereas resilience refers to applying these strategies to succeed in overcoming stress. Thus, while these concepts are related, the general view is that stress coping and resilience are two different dimensions [13].

This study demonstrated that depressed college students are likely to engage in emotion-focused coping and have low resilience for stress. Thus, the condition of mental health for college students could be improved by reducing emotion-focused coping and fostering resilience, after screening students for depression using both internal and external resources within colleges. Programs would be helpful to include cognitive training, mindfulness training, control attention, enhance positive emotions, and mentors to reinforce support system $[32,36,37]$.

This study has the following limitations. First, it included college students from regional communities alone, and the findings cannot be generalized to all college students across the country. Nevertheless, this limitation is partially mitigated by the fact that the study included more than 3,000 participants. Second, the study used self-reported questionnaires, which may have resulted in a subjective bias. A meta-analysis reported that the CES-D, which was used to examine depressive symptoms in this study, tends to yield higher scores compared to depressive symptoms assessed using the BDI [2]. Thus, additional research is necessary to examine the prevalence of depression in college students using several different types of screening tests. Third, cross-sectional studies cannot fully account for the causal relationship between depression and stress coping, or depression and resilience. Future research would be needed to examine closely about the relationship. Lastly, the scales used in this study may also be a limitation. The SCS and BRS have only been used in a handful of studies in Korea to date, and it is difficult to define the characteristics of stress coping and resilience. It is imperative to identify them thoroughly by conducting multiple studies of stress coping strategy and resilience.

\section{CONCLUSION}

This study examined the prevalence of depression, stress coping strategies, and resilience in 3,306 college students in Gwangju and Jeollanam-do regions. These college students reported a high prevalence of depressive symptoms $(12.8 \%)$ with emotion-focused coping and low resilience. As reports indicate increasing prevalence of depressive symptoms among college students every year, it is necessary to pay close attention to the mental health of college students along with improving attempts to provide interventions to enhance stress coping strategies and resilience.

\section{CONFLICTS OF INTEREST}

The authors have nothing to disclose.

\section{ORCID}

Dahni Kim
https://orcid.org/0000-0002-3014-9469
Bo-Hyun Yoon
https://orcid.org/0000-0002-3882-7930
Young-Hwa Sea
https://orcid.org/0000-0002-0420-2609
Hangoeunbi Kang
https://orcid.org/0000-0002-7106-2757


Kyungmin Kim

https://orcid.org/0000-0001-5376-1418

Jye-Heon Song

https://orcid.org/0000-0001-7370-519X

Suhee Park

https://orcid.org/0000-0002-7366-8226

\section{REFERENCES}

1. American Psychiatric Association. Diagnostic and statistical manual of mental disorders: DSM-5. 5th ed. Arlington: American Psychiatric Association; 2013.

2. Ibrahim AK, Kelly SJ, Adams CE, Glazebrook C. A systematic review of studies of depression prevalence in university students. J Psychiatr Res 2013;47:391-400.

3. Sadock BJ, Sadock VA, Ruiz P, Kaplan HI. Kaplan \& Sadock's synopsis of psychiatry: behavioral sciences/clinical psychiatry. 11th ed. Philadelphia: Lippincott Williams \& Wilkins; 2016.

4. Park JH, Min HY, Kim MD, Yoon BH, Bahk WM, Bae HE. The prevalence of depressive symptoms and related factors among college students on Jeju Island in Korea. Mood Emot 2013;11:144-9.

5. Lee ME, Kim SH, Ha KS, Park H, Jung HS, Park JC, et al. Prevalence and factors related to depressive symptoms among university students: from the Honam District, Korea. J Korean Neuropsychiatr Assoc 2016;55:215-23.

6. Healthy Minds Network. National data report, Fall 2020 [Internet]. Michigan: Healthy Minds Network; 2021 [cited 2021 Aug 28]. Available from: https://healthymindsnetwork.org/.

7. Myung JY. Gave up for corona quarantine 'Psychological quarantine' of college students [Internet]. Seoul: Maeil Business Newspaper; 2021 Apr 11 [cited 2021 Aug 28]. Available from: https://www.mk.co.kr/news/society/ view/2021/04/345268/.

8. Orzechowska A, Zajączkowska M, Talarowska M, Gałecki P. Depression and ways of coping with stress: a preliminary study. Med Sci Monit 2013;19:1050-6.

9. Lazarus RS, Folkman S. Stress, appraisal, and coping. New York: Springer Publishing Company; 1984.

10. Park JY. A study on the relationship among character type A \& B ego-identity and stress coping [Master's thesis]. Seoul: Sookmyung Women's University; 1995.

11. Kim JH. A study on factors for stress coping strategies. Korean J Psychol 1985;1985:37-43.

12. Masten AS, Best KM, Garmezy N. Resilience and development: contributions from the study of children who overcome adversity. Dev Psychopathol 1990;2:425-44.

13. Fava GA, Tomba E. Increasing psychological well-being and resilience by psychotherapeutic methods. J Pers 2009;77:1903-34.

14. Rosen JA, Glennie EJ, Dalton BW, Lennon JM, Bozick
RN. Noncognitive skills in the classroom: new perspectives on educational research. North Carolina: RTI Press; 2010.

15. Cho MJ, Kim KH. Use of the Center for Epidemiologic Studies Depression (CES-D) Scale in Korea. J Nerv Ment Dis 1998;186:304-10.

16. Smith BW, Dalen J, Wiggins K, Tooley E, Christopher P, Bernard J. The brief resilience scale: assessing the ability to bounce back. Int J Behav Med 2008;15:194-200.

17. Jung YE, Chae JH. A review of resilience assessment tools. J Korean Neuropsychiatr Assoc 2010;49:50-7.

18. Vandivort DS, Locke BZ. Suicide ideation: its relation to depression, suicide and suicide attempt. Suicide Life Threat Behav 1979;9:205-18.

19. Ministry of Health and Welfare. 2016 The survey of mental disorders in Korea [Internet]. Sejong: Ministry of Health and Welfare; 2017 [cited 2021 Aug 28]. Available from: http://www.mohw.go.kr/react/jb/sjb030301vw. jsp?PAR_MENU_ID $=03 \& M E N U_{-}$ID $=032901 \& C O N T$. $\mathrm{SEQ}=339138 \&$ page $=1$.

20. Lyubomirsky S, Kasri F, Zehm K. Dysphoric rumination impairs concentration on academic tasks. Cognit Ther Res 2003;27:309-30.

21. Cuijpers P, Cristea IA, Ebert DD, Koot HM, Auerbach RP, Bruffaerts R, et al. Psychological treatment of depression in college students: a metaanalysis. Depress Anxiety 2016;33:400-14.

22. Seligman MEP. Helplessness: on depression, development, and death. New York: Freeman; 1992.

23. Joffe H, Cohen LS. Estrogen, serotonin, and mood disturbance: where is the therapeutic bridge? Biol Psychiatry 1998;44:798-811.

24. Lee YM, Pak SY, Kim MJ. Economic stress, depression, suicidal ideation, resilience, and social support in college students. J Korean Acad Psychiatr Ment Health Nurs 2017;26:151-62.

25. Freeman A, Tyrovolas S, Koyanagi A, Chatterji S, Leonardi $\mathrm{M}$, Ayuso-Mateos JL, et al. The role of socio-economic status in depression: results from the COURAGE (aging survey in Europe). BMC Public Health 2016;16:1098.

26. Ministry of Health and Welfare; Korea Foundation for Suicide Prevention. 2021 White paper on suicide prevention [Internet]. Sejong, Seoul: Ministry of Health and Welfare; Korea Foundation for Suicide Prevention; 2021 [cited 2021 Aug 28]. Available from: http:// www.mohw.go.kr/react/al/sal0301vw.jsp?PAR_MENU_ ID $=04 \& M E N U \_I D=0403 \& B O A R D \_I D=140 \& B O A R D$ FLAG $=00 \& C O N T \_S E Q=366297$.

27. Lee HM, Park GE, Hyun YR, Kim YG, Byun HN. "Pay attention to the death of women in their 20s" 12 local legislators speaking in relay [Internet]. Seoul: Hankook Ilbo; 2021 Jul 22 [cited 2021 Aug 28]. Available from: https://www. hankookilbo.com/News/Read/A2021072010040003335.

28. Garlow SJ, Rosenberg J, Moore JD, Haas AP, Koestner B, Hendin H, et al. Depression, desperation, and suicidal ideation in college students: results from the American Foun- 
dation for Suicide Prevention College Screening Project at Emory University. Depress Anxiety 2008;25:482-8.

29. Lee CH, Kim JH. Relations of perceived stress, cognitive set, and coping behaviors to depressidn: a focus on freshmen`s stress experiences. Korean J Couns Psychother 1988;1:25-45.

30. Choi NG, Hegel MT, Sirrianni L, Marinucci ML, Bruce ML. Passive coping response to depressive symptoms among low-income homebound older adults: does it affect depression severity and treatment outcome? Behav Res Ther 2012;50:668-74.

31. Kumpfer KL. Factors and processes contributing to resilience: the resilience framework. In: Glantz MD, Johnson JL, eds. Resilience and development: positive life adaptations. Dordrecht: Kluwer Academic Publishers; 1999. p. 179-48.

32. Southwick SM, Charney DS. The science of resilience: implications for the prevention and treatment of depression.
Science 2012;338:79-82.

33. Kang NW, Kim JH. The correlation among resilience, stressors and stress coping styles. TACR 2012;2:69-94.

34. Park YS, Jang SH. The effects of emotional intelligence, resilience, and communication style on stress coping in college students. J Korea Contents Assoc 2020;20:555-66.

35. Kim MK. Influence of stress coping ability, resilience on nursing performance [Master's thesis]. Seoul: Seoul National University; 2017.

36. Hong Y, Lee J, Lee HJ, Kim K, Cho IK, Ahn MH, et al. Resilience and work-related stress may affect depressive symptoms in nursing professionals during the COVID-19 pandemic era. Psychiatry Investig 2021;18:357-63.

37. Shin YC, Kim SM, Kim H, Min KJ, Yoo SK, Kim EJ, et al. Resilience as a protective factor for depressive mood and anxiety among Korean employees. J Korean Med Sci 2019;34:e188. 